\title{
Prostate Cancer Detection and Diagnosis: Role of Ultrasound with MRI Correlates
}

\author{
Joy Liau' ${ }^{1}$ Daniel Goldberg' ${ }^{1}$ Hina Arif-Tiwari ${ }^{1}$
}

Published online: 7 February 2019

(C) The Author(s) 2019

\begin{abstract}
Purpose of Review Prostate cancer (PCA) detection and diagnosis has rapidly changed in recent years, with medical imaging playing an instrumental component. Multiparametric magnetic resonance imaging (MRI) has led to an alternative diagnostic pathway for patient triage and diagnosis. Ultrasound (US) is already essential to this pathway, and development of new US technologies ensures it will assume an even more important role.

Recent Findings Conventional US is widely used for anatomic guidance during prostate biopsy, but has low PCA detection rates. Ultrasound elastography and contrastenhanced US are promising for improving PCA detection, have been utilized in addition to traditional systemic biopsy, and provide complementary information to MRI.

Summary Here we summarize the current clinical use and newest developments in prostate US. We also present their MRI correlates with the vision that the two modalities will be used closely together in the future to improve PCA care.
\end{abstract}

Keywords Prostate cancer · Ultrasound · Ultrasound elastography $\cdot$ MRI $\cdot$ Detection $\cdot$ Diagnosis

This article is part of the Topical Collection on Ultrasound.

Joy Liau

jliau@email.arizona.edu

Daniel Goldberg

dgoldberg@radiology.arizona.edu

Hina Arif-Tiwari

hinaarif@radiology.arizona.edu

1 Department of Radiology, University of Arizona, $1501 \mathrm{~N}$ Campbell Avenue, Tucson, AZ 85724, USA

\section{Introduction}

Prostate cancer (PCA) is second only to lung cancer as a leading cause of death and has the highest cancer incidence in United States men [1]. Increased use of prostate-specific antigen (PSA) testing led to a dramatic increase in PCA incidence in the late 1980s and early 1990s, and the resulting early detection from PSA testing and improved treatment caused a decline in the PCA death rate by $52 \%$ from 1993 to 2015 [1]. The management of PCA continues to rapidly change, with both the ultrasound (US) and magnetic resonance imaging (MRI) modalities becoming indispensable components in detection and diagnosis.

In the traditional diagnostic pathway, clinical suspicion for PCA is raised with abnormalities in the digital rectal exam (DRE) and/or an elevated PSA [2•, 3]. Then, a transrectal ultrasound (TRUS)-guided systemic biopsy (SBx) is performed, which involves taking 10-12 biopsy cores equally distributed throughout both sides of the prostate using conventional US for anatomic guidance but

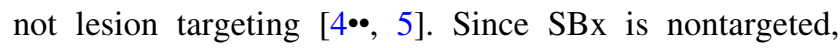
20-30\% of clinically significant PCAs may be missed on first biopsy [4*0]. Even when PCA is detected, SBx may under-stage disease when it does not sample the most aggressive portion of the lesion [6]. SBx also leads to overdiagnosis of PCA by sampling clinically insignificant cancers. It is now understood that lower grade (clinically insignificant) PCAs (total Gleason score $\leq 6$ ) have much lower mortality then clinically significant PCAs (total Gleason score $\geq 7$ ), with $4+3$ having triple the mortality of $3+4$ disease [7]. However, despite evidence from randomized clinical trials that treatment of clinically insignificant PCA is not beneficial, diagnosis often leads to overtreatment or active surveillance, both of which are 
expensive and can negatively impact the patient's quality of life [3].

In recent years, development of an alternative diagnostic pathway involving multiparametric magnetic resonance imaging (mpMRI) promises to revolutionize PCA management. In this pathway, once clinical suspicion is raised, the patient undergoes mpMRI of the prostate as part of a triage process. Biopsy can then be avoided with negative mpMRI results, and targeted biopsy may be done with positive mpMRI. Use of MRI-targeted biopsy improves detection of clinically significant PCAs compared to SBx (sensitivity $91 \%$ vs. $76 \%$ ) and decreases detection of insignificant PCAs (sensitivity $44 \%$ vs. $83 \%$ ) [8]. MRItargeted biopsy may be done via transperineal or transrectal approach by three methods (MRI in-bore, cognitive fusion, and MRI-TRUS fusion), the latter two methods also utilizing US. With the MRI in-bore method, serial MRIs are used to track the biopsy needle placement, making it the only method which allows real-time visualization of the MRI localized lesion during biopsy. However, this method is not often used since it requires MRI scanner time and special MRI-compatible equipment, and is a time-intensive procedure which may be uncomfortable for the patient who is required to be in the prone position [5]. In cognitive fusion, the clinician mentally localizes the target while performing TRUS biopsy under conventional US guidance. Since the lesion is not visualized in real-time, this can be a subjective procedure [9]. MRI-TRUS fusion is a widely used technique where the target lesion is marked on the prebiopsy mpMRI and fused via software to the real-time TRUS gray-scale (B-mode) images. A spatially tracked US transducer then allows localization with the fusion images during the biopsy.

With MRI-targeted biopsy, a clinical dilemma is presented when negative biopsy results are obtained after a positive mpMRI. False-positive mpMRI findings may be caused by benign prostatic hyperplasia, prostatitis, and high-grade intraepithelial neoplasia [10•]. However, a large number (10-15\%) of clinically significant PCAs are missed by mpMRI and targeted biopsy [10॰]. It is not clear which misses are due to insensitivity of mpMRI, or errors in sampling when the lesion is not visualized in real-time with TRUS. Major sources of error with MRI-TRUS fusion biopsy include co-registration and performance errors. Coregistration of the MRI and US images is degraded when the axial MRI and US slices are acquired in slightly different planes and with differences in prostate shape deformation due to patient orientation, bladder and rectal filling, and pressure effects of the endorectal coil or TRUS probe $\left[9,10^{\bullet}\right]$. Also, performance of MRI-TRUS fusion biopsy improves with experience, with greater experience resulting in increased detection rates and decreased Gleason score upgrading at the time of prostatectomy [10॰].
Currently, discrepancies in mpMRI reads and biopsy results may be managed by PSA monitoring, repeat mpMRI, or repeat biopsy as dictated by degree of clinical suspicion [10•].

The new diagnostic pathway using mpMRI to triage and target biopsies is clearly advantageous to SBx. Additional improvements need to be made to reduce the number of clinically significant cancers missed by this pathway and errors that may result from using TRUS guidance to sample lesions identified on MRI. Conventional US is already integral to the most common applications of this pathway, but additional US modalities have a complementary role to MRI and may improve PCA management when used together. Here, we summarize the role of US in PCA detection and diagnosis and present their MRI correlates.

\section{Conventional Ultrasound}

Conventional US methods of B-mode and Doppler via TRUS are currently the most commonly used methods to guide prostate biopsies. These modalities are widely available due to their low cost, real-time performance, and lack of ionizing radiation $[4 \bullet \bullet]$.

\section{B-mode Ultrasound}

B-mode US is primarily used to depict anatomy. The prostate is scanned in transverse and sagittal planes including the entire gland from apex to base. This allows measurement of prostate volume and identification of normal zonal anatomy. In the normal prostate gland, the outer peripheral zone is homogenous and slightly more echogenic than the inner central or transitional zones (Fig. 1a) [9]. Areas of abnormal echogenicity such as calcifications (Fig. 1b) and especially hypoechoic lesions suspicious for PCA (Fig. 1c) may be identified.

Although PCAs can sometimes be identified on B-mode US as hypoechoic lesions, B-mode US alone is not adequate for detection or staging of PCA. One study including 30 patients evaluated by B-mode US and prostatectomy found that out of 29 hypoechoic lesions found in the peripheral zone, $22(76 \%)$ were PCAs and 7 (24\%) were false positives; a total of 40 cancers $(64.5 \%)$ were missed on B-mode imaging [11]. The B-mode US appearance of focal lesions is nonspecific because nonmalignant conditions such as prostatitis and benign prostatic hyperplasia may be hypoechoic, and early-stage PCA with a greater fraction of normal glandular tissue can be isoechoic [9]. Disease staging should also not rely on B-mode US, which has low sensitivity although high specificity for extracapsular extension (sens $11.8 \%$, spec $96 \%$ ) and seminal vesicle invasion (sens 9.8\%, spec 99\%) [12]. 

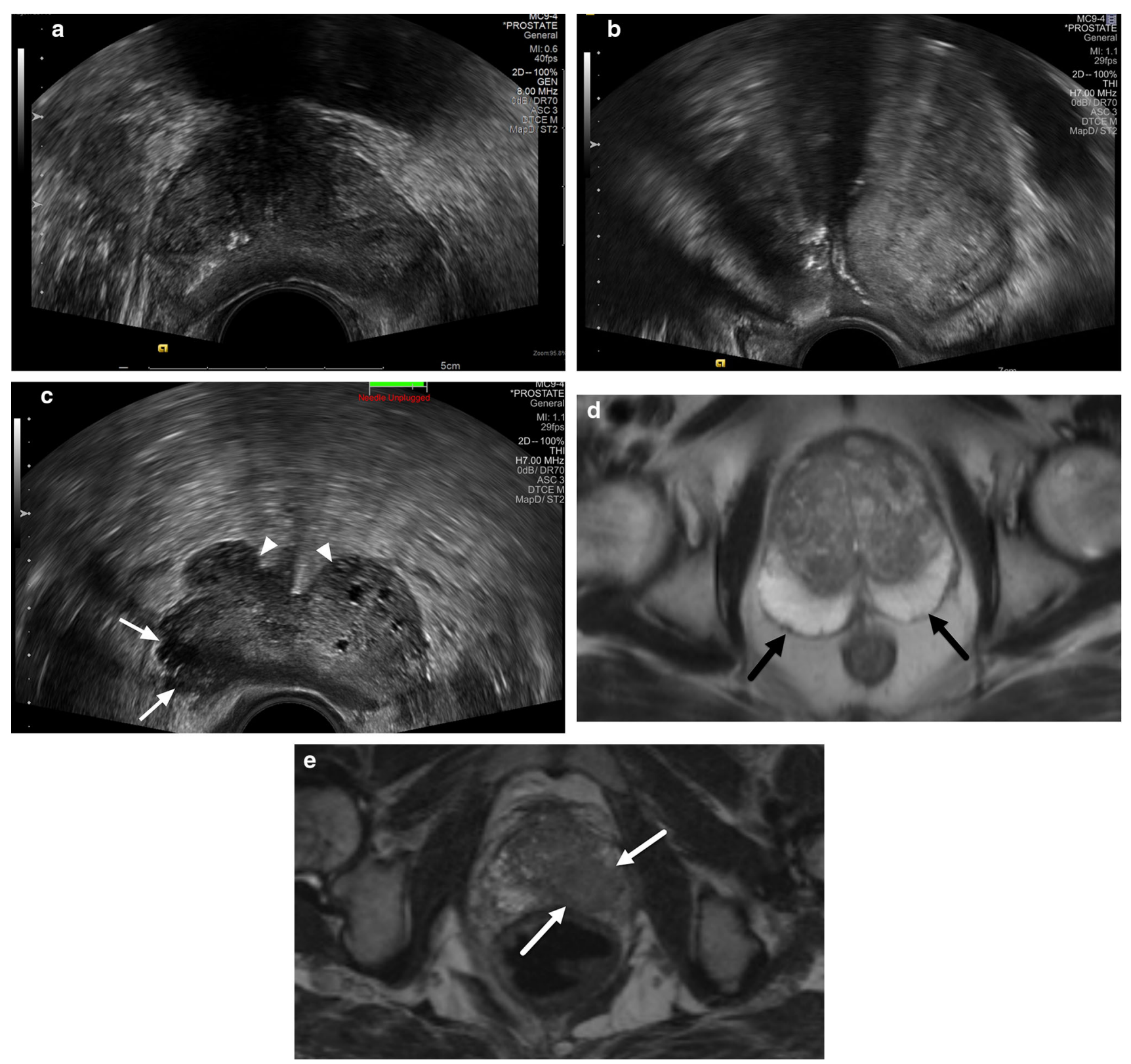

Fig. 1 a B-mode US image of normal prostate: homogenous peripheral zone of the prostate gland is seen abutting the rectal wall on B-mode transrectal sonogram. Central gland shows heterogeneity due to changes related to benign prostatic hyperplasia including nonshadowing calcifications at the junction of right central and peripheral gland. b B-mode US prostate calcifications: transrectal sonogram shows diffuse prostatomegaly in another patient, with marked enlargement of the central gland. Shadowing periurethral and right gland calcifications are seen which obscure the anterior portion of the prostate gland. c B-mode US image of prostate cancer: the

The MRI correlate for B-mode US is T2 weighted (T2w) imaging. High-resolution axial T2w MRI is performed in mpMRI to show normal prostate anatomy, identify suspicious lesions, and stage disease. The normal peripheral zone of the prostate is high in water content due to its abundance of ductal and acinar components, resulting in hypoechoic prostate cancer (arrows) is present in the right posterior peripheral zone at the level of the mid-gland. Transurethral resection of the prostate changes are present (arrowheads). d T2w MRI normal prostate: High-resolution $\mathrm{T} 2 \mathrm{w}$ image of the prostate gland shows normal homogenous hyperintense peripheral zone of the prostate gland (arrows). e T2w MRI prostate cancer: PCA appears hypointense on $\mathrm{T} 2 \mathrm{w}$ image (arrows), on background on bright hyperintense peripheral zone, that provides good inherent tissue contrast for lesion identification

high T2 signal. The normal transitional zone has more smooth muscle and less glandular content, reflected as lower T2 signal (Fig. 1d) [9]. PCA appears as a round or ill-defined T2 hypointense lesion on MRI (Fig. 1e). Low T2 signal on MRI alone is not specific for cancer however, 

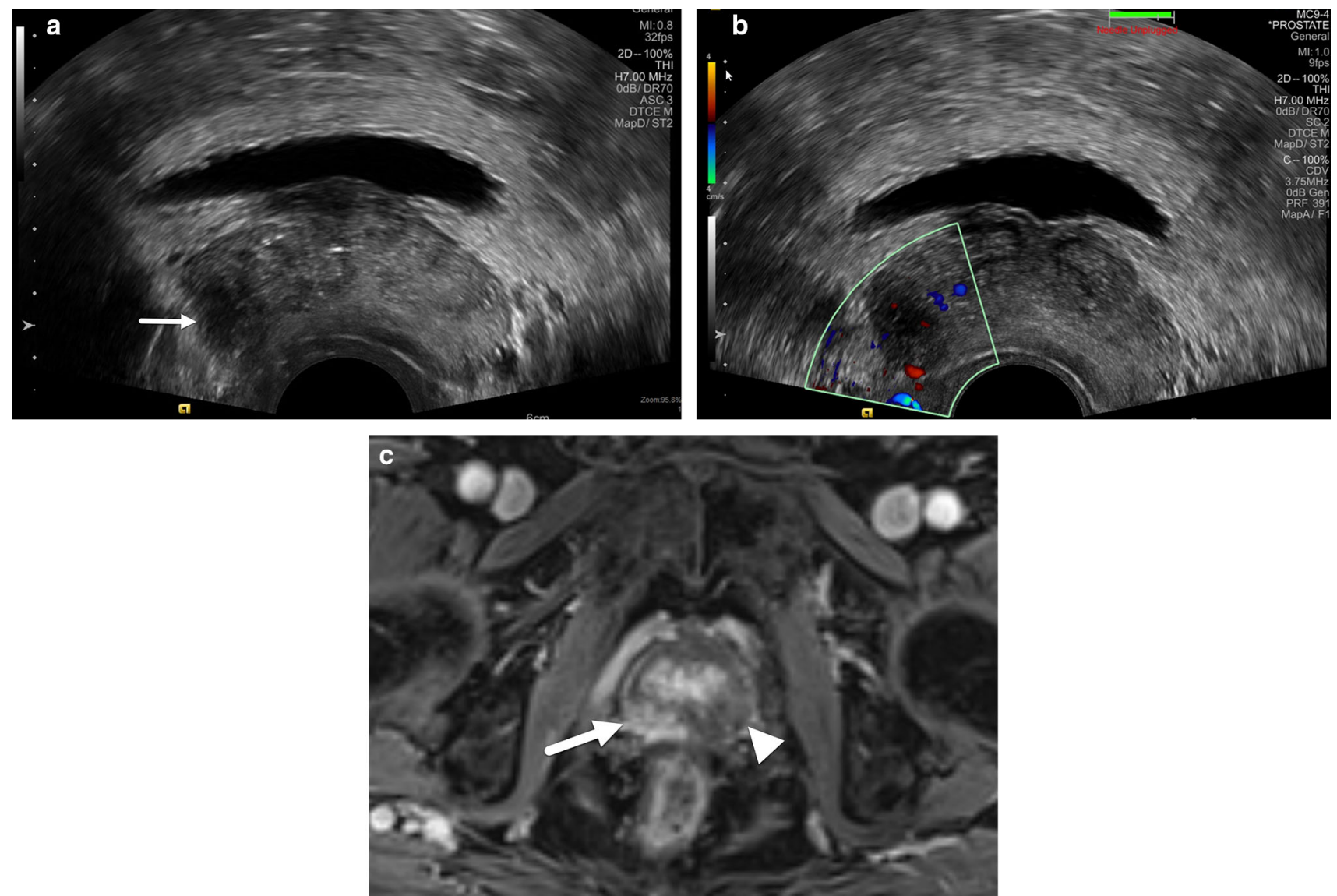

Fig. 2 a B-mode and b Doppler US image of prostate cancer: B-mode image on transrectal sonogram (a) depicts a well-defined heterogeneously hypoechoic mass in the right peripheral zone at the level of the mid-gland (arrow). b Duplex evaluation of the tumor shows mild internal and peripheral vascularity due to tumor neoangiogenesis. c DCE-MRI arterial phase prostate cancer: gradient

as T2 hypointensity is seen with prostatitis, atrophy, and benign prostatic hyperplasia in the transitional zone [9].

\section{Doppler Ultrasound}

Doppler US is used as needed in prostate TRUS to detect and characterize focal lesions. PCA growth results in angiogenesis and increased microvessel density [13]. In color Doppler US, frequency shifts from US waves reflecting off moving red blood cells are proportional to blood flow velocity, revealing areas of increased perfusion which can be seen with PCA (Fig. 2a, b). Power Doppler US shows the integrated power of the Doppler signal, increasing sensitivity. Since Doppler US has limited ability to detect blood flow in vessels less than $0.1 \mathrm{~mm}$ in diameter and much of cancer growth is comprised of microvessels $10-50 \mu \mathrm{m}$ in diameter, Doppler US may only be able to detect the larger macrovessels present in higher grade PCAs [9]. echo T1w image with fat saturation shows avid arterial enhancement within the tumor secondary to neovascularity (arrow). Please note the normal peripheral zone does not show first pass contrast uptake (arrowhead) on dynamic post-gadolinium imaging. Heterogenous contrast uptake in the central gland can be normally seen

There have been mixed results in studies assessing use of Doppler US for PCA detection. One study comparing color and power Doppler US to sextant biopsy found Doppler US was not useful for PCA detection [14], while another study of power Doppler US found a sensitivity of $98 \%$ and specificity of $78 \%$ [15]. Supporting the idea that only higher grade PCAs may be detected by Doppler US, a study including 243 patients evaluated by power Doppler US with targeted and systemic biopsy found poor performance for detecting low-risk PCA (sensitivity 45\%, specificity 74\%), but that a normal power Doppler US had a $96 \%$ chance of the patient not having a high-risk PCA [16].

Dynamic contrast-enhanced MRI (DCE-MRI) is the correlate for Doppler US. In DCE-MRI, serial T1 weighted images are continuously obtained before, during, and after the injection of intravenous gadolinium contrast. It detects abnormal blood flow related to PCA tumor angiogenesis, which is typically characterized by sharp contrast uptake 
peaking $60 \mathrm{~s}$ after bolus administration (Fig. 2c) and rapid washout [17]. Contrast movement between the extravascular, intravascular, and intracellular spaces can be quantitatively measured as $K^{\text {trans }}$ (a measure of vascular permeability, the transfer constant between vascular and intracellular spaces), $K_{\text {ep }}$ (contrast movement back into the vascular space), and $V_{\mathrm{e}}$ (first pass contrast diffusion into the extravascular space) [17].

\section{Ultrasound Elastography}

Ultrasound elastography (USE) assesses tissue elasticity, which is the ability of tissue to resist deformation with application of force, or to return to original shape after cessation of the force [18]. It can also be thought of as tissue stiffness, the same property used by physicians in the DRE to palpate hard PCA nodules. PCA is characterized by angiogenesis, increased cellular density, and destruction of glandular architecture, which triggers wound repair mechanisms and produces stromal reaction and collagen deposition, increasing tissue stiffness [19]. USE is performed after conventional US to detect and characterize PCAs. There are two major methods of prostate USE: (1) strain elastography (SE), and (2) shear wave elastography (SWE).

\section{Strain Elastography}

Physics In SE, the clinician manually compresses the prostate using the ultrasound transducer, exerting stress on the tissue. The resulting tissue displacement in the same direction as the applied stress allows an estimate of tissue strain $\varepsilon$ (Fig. 3). The tissue displacement is measured via

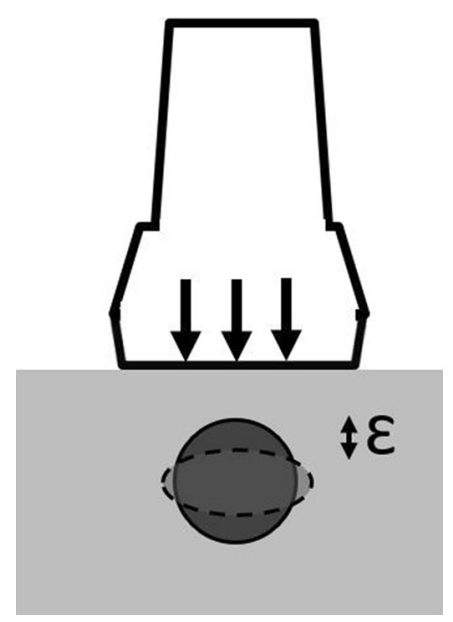

Fig. 3 Strain elastography diagram: the clinician exerts force on the ultrasound transducer to compress the tissue (arrows). The lesion of interest (solid circle) is deformed by the compression (dash ellipse), with the measured tissue displacement $(\varepsilon)$ reflecting lesion elasticity ultrasound methods such as radiofrequency echo correlation-based tracking and/or Doppler processing [18]. This is not a quantitative method since the manually applied stress cannot be measured. However, by assuming uniform stress $\sigma$ application and lack of time dependent deformation (viscosity), the measured strain $\varepsilon$ allows a qualitative measure of Young's Modulus $(E)$ and tissue elasticity via Hooke's Law $(\sigma=E \cdot \varepsilon)$ [18].

Technique To perform SE, the patient assumes a left lateral position or lithotomy position. The ultrasound transducer is covered with coupling gel and slowly inserted into the rectum. The clinician applies continuous small compressions and releases which are assessed by the system's quality index for appropriateness [4••]. To improve uniform application of stress on the prostate gland, a waterfilled balloon may be placed between the transducer and rectal wall [20].

Image Interpretation Strain measurements are displayed on a color map referred to as an elastogram and overlaid on the B-mode image. Stiff tissues (low strain) are commonly shown as blue and soft tissues (high strain) are shown as red, although color scales can vary by manufacturer [4••, 18]. The normal prostate peripheral zone is of intermediate stiffness. The central gland (transition and central zones) has a homogenous soft pattern in young men, and increase in volume and stiffness with age $[4 \bullet \bullet$. The prostate capsule has a normal pericapsular "soft rim artifact" which can be lost with extracapsular extension of PCA [21], a highly specific $(96 \%)$ but poorly sensitive (38\%) finding [22].

The qualitative data of elastograms can be assessed semi-quantitatively by application of scoring systems. For example, a 5-point scoring system used by Kamoi et al. assigns a higher score and increased probability of malignancy to stiff and asymmetric lesions which are hypoechoic on B-mode US, producing a sensitivity of $68 \%$ and specificity of $81 \%$ with a cut-off value of 3 [23]. Xu et al.'s system also has a 5-point score, and assesses lesions in the peripheral gland with increased stiffness, asymmetry, and size as malignant features; using a cut-off value of 3 , this system had a sensitivity of $68.6 \%$ and specificity of $69.4 \%$ [24]. Alternatively, a semi-quantitative measurement called the peak strain ratio index can be computed by taking the ratio of the strain measured in the normal prostate tissue to the peak strain measured in the lesion. In a group of 141 patients assessed by SE and biopsy, the peak strain index was significantly higher in malignant than benign lesions (means 24.79 vs. 3.02, $p<0.0001$ ), and using a peak strain index cut-off of $\geq 5.97$ produced a high sensitivity of $87.5 \%$ and specificity of $85.5 \%$ with area under the curve (AUC) of 0.95 [25]. 
Review of Literature Multiple studies have assessed the use of SE for PCA detection. SE has better performance than B-mode US for PCA evaluation in the peripheral zone, with sensitivity, specificity, negative predictive value (NPV), and positive predictive value (PPV) of 48, 81, 58, and $75 \%$ with B-mode and 66, 78, 67, and $77 \%$ with SE [26]. Comparisons of SE targeted-biopsy and SBx generally show increased PCA detection with addition of SE. A prospective study of 353 patients showed improved detection with SE targeted-biopsy (51.1\%) compared to $\operatorname{Sbx}(39.4 \%, p=0.027)$ [27]. Another prospective study of 230 patients showed similar improvement of detection with SE guidance $(30 \%)$ compared to SBx $(25 \%)$ [28]. Wang et al.'s assessment of 108 patients using transrectal SE guidance with transperineal targeted-biopsy and SBx found a $13.9 \%$ increase in PCA detection with addition of SE [29]. A large retrospective study evaluating 1024 patients similarly showed an increased detection rate of $24.8 \%$ by addition of SE targeted-biopsy to SBx in patients undergoing re-biopsy [30]. However, another retrospective study demonstrated low sensitivity (19.8\%) although high specificity (90.9\%) of SE targeted-biopsy in 519 re-biopsy patients [31]. The SE detection rates of PCA may also depend on tumor grade, with increased cell density and thus stiffness of higher grade tumors improving SE sensitivity. Sumara et al. found that PCA detection rates for Gleason scores 6, 7, and 8-9 were 60, 69.2, and 100\%, respectively [32]. Overall, existing literature indicates addition of SE targeted-biopsy to SBx can increase PCA detection rate, but SE should not be used without SBx due to its low sensitivity.

SE may be able to detect PCA lesions not visible on MRI and can be viewed as a complementary technique. Using radical prostatectomy histology as the reference standard, Sumura et al. found detection rates to be superior in SE $(74.1 \%)$ compared to T2 weighted MRI (42.1\%) and DCE-MRI (47.4\%) [32]. Pelzer et al. similarly found improved detection of the dominant PCA lesion by SE (92\%) versus MRI (84\%); the performance was location dependent, with improved SE sensitivity in the apical and middle prostate and better MRI sensitivity in the base and transition zone of the prostate [33]. Combining the two techniques, Brock et al. found that the MRI/SE fusion targeted-biopsy had superior sensitivity and specificity (77.8\% and $77.3 \%$ ) compared to MRI-targeted biopsy $(74.1 \%$ and $62.9 \%)$ in re-biopsy patients [34].

Limitations There are multiple limitations in the use of SE for PCA detection. First, SE is a qualitative technique that assumes uniform prostate compression. Balloon interposition can improve compression uniformity, reducing image artifacts caused by slippage in the compression plane from 32 to $1 \%$ of images in one study [20].
Computational methods using finite element analysis to reconstruct images accounting for stress nonuniformity may also improve results [35]. Another limitation is the variability of SE technique. Performance of SE is operator dependent with a known learning curve, but no consistent training protocol exists [4••]. The color code schemes of elastogram display are not standardized and depend on the ultrasound vendor [18]. The performance of SE is also influenced by factors such as tumor size, location, and prostate volume. PCA detection rates by SE improve with increasing tumor size $(9.7 \% \quad 0-5 \mathrm{~mm}, 27 \% \quad 6-10 \mathrm{~mm}$, $70.6 \% 11-20 \mathrm{~mm}, 100 \%>20 \mathrm{~mm}$ ) [36]. SE has improved sensitivity at the apex of the prostate (60-76.9\%) compared to the base (34.2-45\%) likely due to better application of compression and decompression of small volumes at the apex [27]. Similarly, smaller volume prostates yield higher detection rates than large prostates [24]. Additionally, stiffness is not a feature specific to cancer, but false-positive results may result from benign entities such as prostatitis, prostate calcifications, fibrosis, atrophy, adenomyomatosis, and benign prostatic hyperplasia [37].

\section{Shear Wave Elastography}

Physics SWE is a quantitative method, unlike SE. In contrast to the operator-dependent manual compression of $\mathrm{SE}$, the stimulus in SWE is acoustic radiation force. In this technique, a high-intensity short-duration acoustic "pushing pulse" is applied to a selected focal zone and displaces tissue in the same direction as the ultrasound transducer. A fraction of the generated waves is intra-converted into shear waves through the absorption of acoustic energy, which propagate away from the focal zone in the perpendicular direction [18]. Interrogation of multiple focal zones in rapid succession forms a near cylindrical shear wave cone (Fig. 4), allowing quantitative 2D measurements of shear wave speed [18]. Shear wave speed $\left(c_{\mathrm{s}}\right)$ is related to elasticity or Young's modulus $(E)$ by the equation $E=3 \rho c_{\mathrm{s}}$, where $\rho$ is density. Measurements of shear wave speed in $\mathrm{m} / \mathrm{s}$ or Young's modulus in $\mathrm{kPa}$ are color coded and overlaid on B-mode images to form 2D quantitative elastograms [18].

Technique SWE is performed transrectally after conventional US with the patient in left lateral position. The prostate is scanned throughout the base to apex while avoiding any pressure on the transducer. The transducer is held steady 2-4 s in each plane to allow the signals to stabilize $[4 \bullet \bullet]$.

Image Interpretation Interpretation of $\mathrm{SWE}$ is more straightforward since measurements are quantitative. A region of interest is placed on the lesion to obtain the mean 


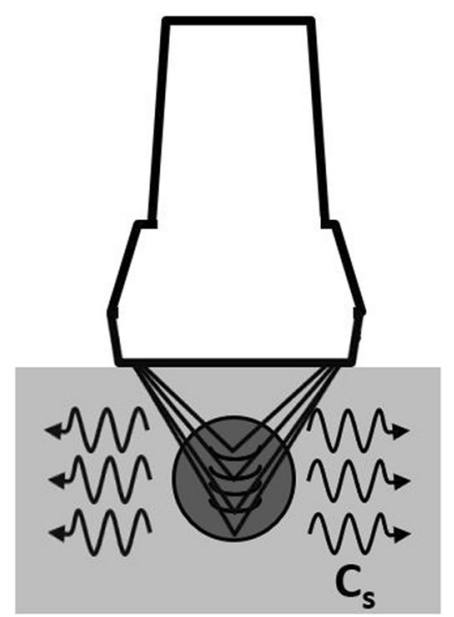

Fig. 4 Shear wave elastography diagram: the clinician holds the ultrasound transducer steady as acoustic radiation force displaces tissue in the lesion of interest (solid circle) in the same direction as the transducer. This produces perpendicularly oriented shear waves with speed $\left(c_{\mathrm{s}}\right)$ directly related to tissue elasticity

stiffness measurement. A SWE ratio may also be obtained by dividing the stiffness in the normal tissue by the stiffness of the lesion $[4 \bullet \bullet, 18]$. Similar to SE, the peripheral zone is soft and homogenous, while the central gland increases in stiffness and heterogeneity with age (Fig. 5a, b) [4••]. PCA is detected as a focal region of increased stiffness (Fig. 5c, d).

Review of Literature Although SWE is a relatively new technique, multiple studies have evaluated its use for PCA detection. In a retrospective study of 87 patients, a stiffness cut-off of $43.9 \mathrm{kPa}$ produced low sensitivity (43\%), but high specificity (80.8\%) with AUC of 0.599 [38]. Correas et al. performed a prospective study in 184 men and found significantly increased stiffness of PCA compared to benign tissue $(p<0.001)$, and a cut-off of $35 \mathrm{kPa}$ yielding a sensitivity, specificity, PPV, and NPV of $96 \%, 85 \%$, $48 \%, 99 \%$ [39]. In a study with 60 patients, Boehm et al. found that PCA $(88 \mathrm{kPa})$ to be stiffer than benign tissue (42 $\mathrm{kPa}, p<0.001)$ and sensitivity, specificity, PPV, and NPV of $80.9,69.1,67.1$, and $82.2 \%$ using a cut-off of $50 \mathrm{kPa}$ [40]. A recent prospective study of 212 men showed that a cut-off of $82.6 \mathrm{kPa}$ had a high sensitivity (96.8\%) with specificity of $67.8 \%$ and AUC of 0.976 [41]. However, Porsch et al. found poor results in a smaller number of patients $(n=10)$ with a cut-off of $50 \mathrm{kPa}$, producing sensitivity, specificity, and AUC of 74\%, 43\%, and 0.527 [42]. Only one study has compared SWE targeted-biopsy to SBx; they found that although the per-core detection rate was not significantly different $(10.5 \%$ vs. $8.6 \% p=0.3$ ), suspicious SWE findings had a 6.4 times greater chance of clinically significant PCA $(p=0.03)$ [43]. Although results are mixed, overall SWE appears to increase PCA detection and may be useful in addition to SBx.

Similar to SE, SWE sensitivity may increase with higher grade PCA. Woo et al. found significant linear correlations between Gleason score and mean stiffness $(r=0.343$, $p=0.002)$ and mean stiffness ratio $(r=0.296, p=0.008)$ [38]. A study by Wei et al. showed increasing stiffness by tumor grade $(91.6,102.3$, and $131.8 \mathrm{kPa}$ for Gleason scores 6,7 , and $\geq 8$ ) with benign tissue measuring $58.3 \mathrm{kPa}$ [41].

Limitations Although SWE benefits by being a quantitative technique, there are a number of limitations. There is still a learning curve present for SWE, albeit smaller than for SE, but no standardized training procedure exists [4••]. Excess pressure from the transducer can exaggerate stiffness measurements, and can be difficult to avoid with large prostates that protrude into the rectum [4••]. Furthermore, stiffness of normal prostate tissue is technique and location dependent. Benign tissue in the transitional zone is stiffer than in the peripheral zone. Stiffness measurements in the normal medial peripheral zone are higher than in the lateral peripheral zone, which may be due to proximity to and pressure from the ultrasound probe $[11,38]$. Measurements taken in the sagittal plane are stiffer than the axial plane, possibly due to a combination of differences in transducer pressure and tissue anisotropy [11]. These locational differences in stiffness of benign tissue may require different cut-off values to correctly identify malignancy, notably in the transitional zone. A technical challenge of SWE is the acoustic radiation force used for imaging can only penetrate 3-4 cm of depth, limiting assessment of the anterior gland of large prostates (Fig. 6a, b) [4••, 11]. Finally, similar to SE, several benign processes also cause stiff tissue and can be difficult to distinguish from PCA [37]. For example, prostate calcifications cause high stiffness measurements (Fig. 6c, d), but can be identified on B-mode US.

\section{MRI Correlate of Ultrasound Elastography}

The MRI correlate for USE is diffusion-weighted imaging (DWI). In DWI, bipolar magnetic field gradients are applied to sensitize the MRI signal to water motion in tissues. The increased cellular density that causes tissue stiffness in PCA assessed by USE also restricts Brownian motion of water molecules in DWI [9, 17]. Two or more sets of DWI images are typically acquired with varying degrees of diffusion weighting ( $b$-value). The relatively restricted diffusion of water molecules in PCA compared to surrounding normal tissue results in increased signal in the lesion on DWI, visualized as decreased signal when the image is inverted (Fig. 7a). The acquisition of multiple $b$ values allows measurement of the exponential signal decay 

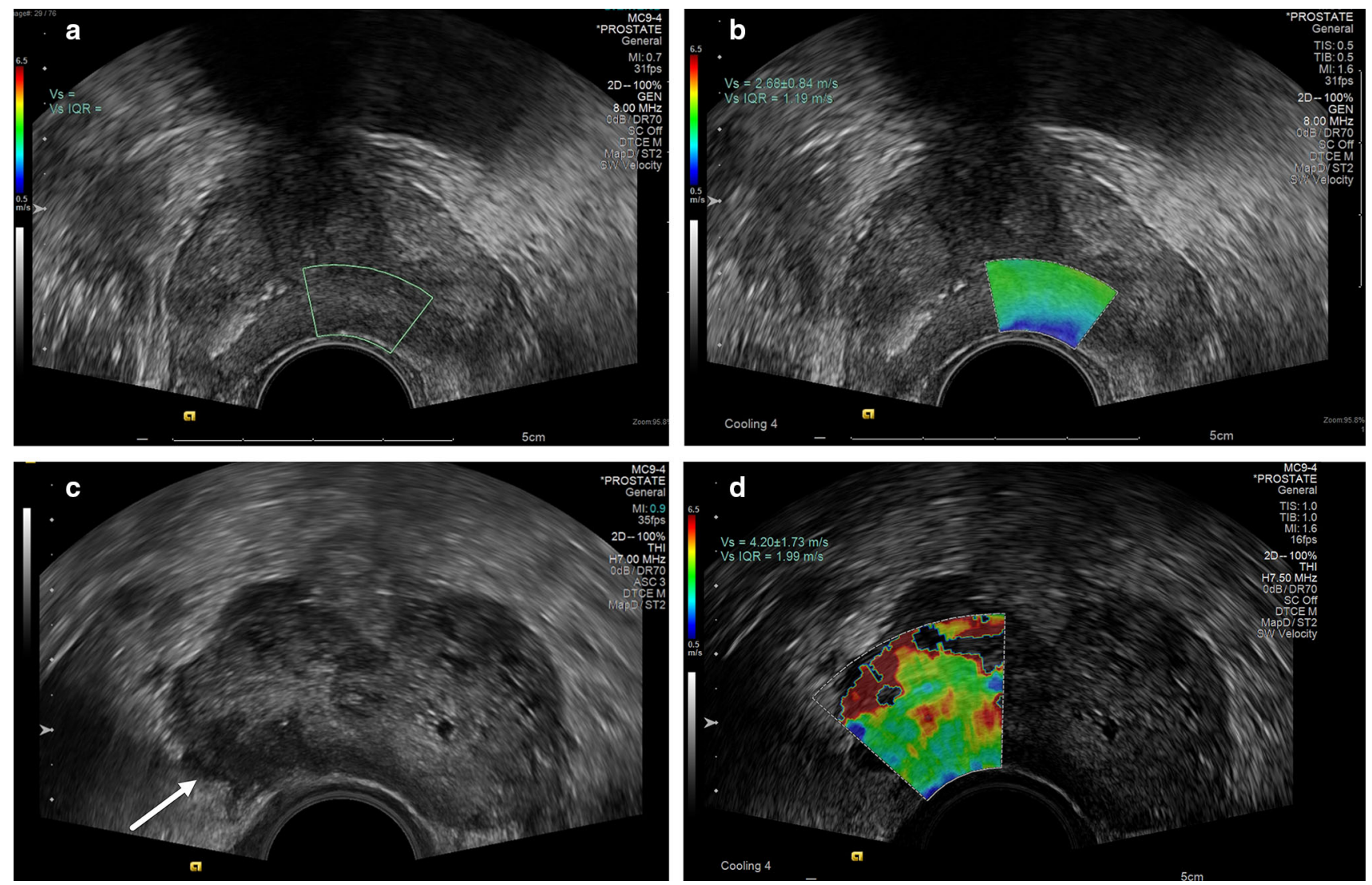

Fig. 5 a B-mode and b SWE US of normal prostate: Transrectal sonogram shows B-mode (a) and SWE (b) through the peripheral zone, which appears homogenous on the gray-scale image. On application of SWE, high strain (soft) tissue characteristics are revealed in the peripheral zone (blue), which transitions to stiffer tissue in the central gland (green) on the color overlay map. c B-mode

with increasing diffusion weighting; this is used to compute the quantitative apparent diffusion coefficient (ADC), which appears as low signal intensity with PCA (Fig. 7b) [9].

\section{Contrast-Enhanced Ultrasound}

In contrast-enhanced ultrasound (CEUS), gas-filled microbubbles with phospholipid or protein shells approximately the size of red blood cells are injected intravascularly. These microbubbles are too large to cross the endothelium of blood vessels and remain within the intravascular space. Application of ultrasound pressure causes nonlinear oscillations of the bubbles which backscatter US signal at harmonics of the incident frequency. Extraction of the nonlinear signals allows dynamic measurement of tissue perfusion [9, 44, 45]. Tumor angiogenesis in PCA produces increased microvascular density which can be measured by CEUS. Suspicious areas in the prostate are asymmetric and have rapid or relatively increased contrast enhancement [46].

and $\mathbf{d}$ SWE US of PCA. B-mode image on transrectal US shows an irregularly shaped hypoechoic mass in the right peripheral zone at the level of mid-gland. SWE US through the tumor depicts heterogeneously increased tissue stiffness demonstrated as red areas with high shear wave velocity measurement and low tissue strain (Color figure online)

Studies have been overall promising in the use of CEUS for PCA detection. $\mathrm{Li}$ et al. performed a large meta-analysis of 16 studies including 2624 patients assessing CEUS in PCA and found a pooled sensitivity and specificity of $70 \%$ and $74 \%$ [47]. However, one study found no significant difference in PCA detection rate based on core number between CEUS targeted-biopsy (18.8\%) and SBx (18.3\%) in a relatively small population of 26 patients [46]. In contrast, a recent large prospective study with 1024 patients found CEUS targeted-biopsy improved detection of clinically significant PCA compared to Sbx $(28.7 \%$ vs 25.3\% $p=0.000$ ) [45]. Although studies suggest that CEUS may improve detection of PCA, the relatively low sensitivity only warrants its use in conjunction with SBx.

Multiple factors limit the application of CEUS to PCA management. Compared to the other US modalities discussed above, CEUS is more invasive because it requires injection of microbubble contrast agent via an IV line. There is a limited time window due to contrast bolus dynamics to scan the entire prostate or perform targetedbiopsy in CEUS. Also, CEUS is difficult to quantify 

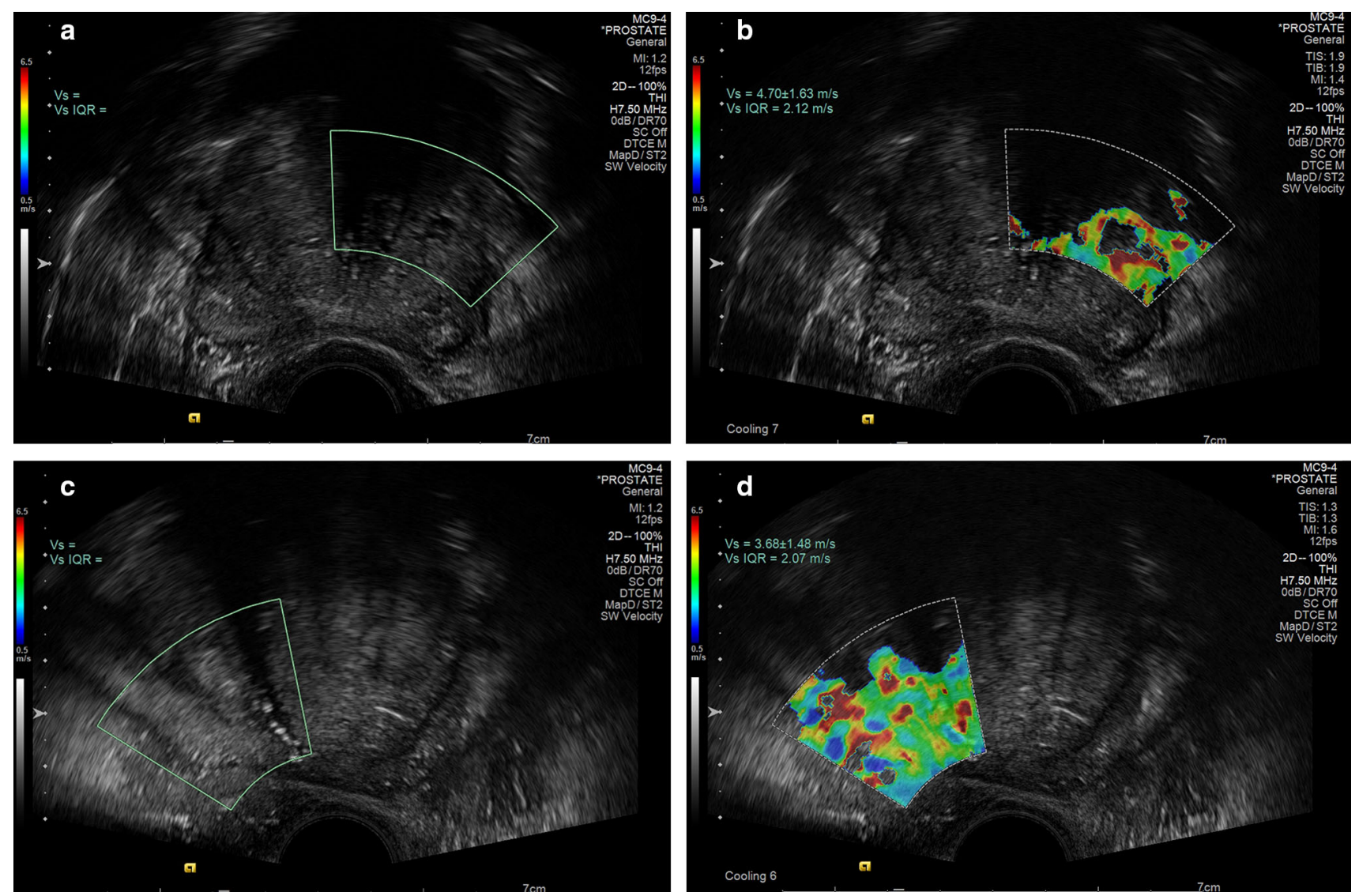

Fig. 6 a B-mode and b SWE US depth limitation: Interrogation of anterior prostate parenchyma, as shown on B-mode image (a) is challenging on SWE US (b), due to deficient compression of the tissues within the anterior gland; this results in a poor-quality elastogram with areas of signal loss in the left lateral anterior prostate. Of note, the midline anterior prostate has shadowing artifact, resulting

in signal loss on both B-mode and SWE images. c B-mode and d SWE US calcification-related artifact: Calcifications with the prostate parenchyma (a) may result in increasing tissue stiffness, and thereby artifactual demonstration of high shear wave velocities and low strain values, shown as red areas on the SWE image (d) (Color figure online)

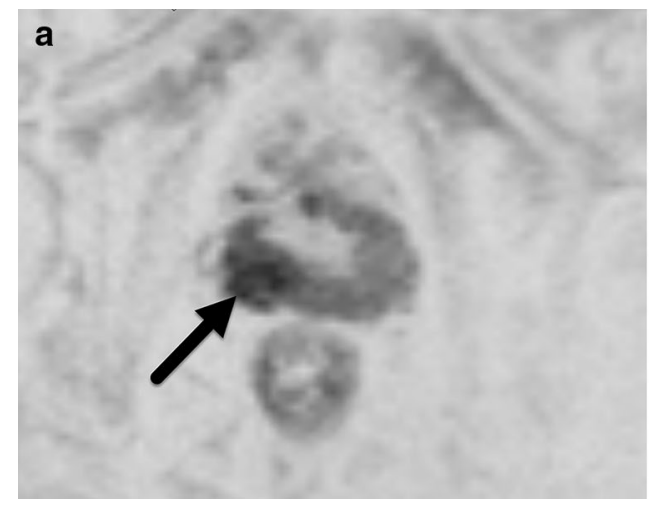

Fig. 7 a PCA DWI, high $b$-value: increased restriction of water molecule diffusion across the cell membrane within the tumor is shown as a dark area (arrow) on this high $b$-value image, which is

because the signal is affected by differences in scanner settings, bubble properties and handling, and patient factors which may change microbubble circulation or attenuation effects [44].

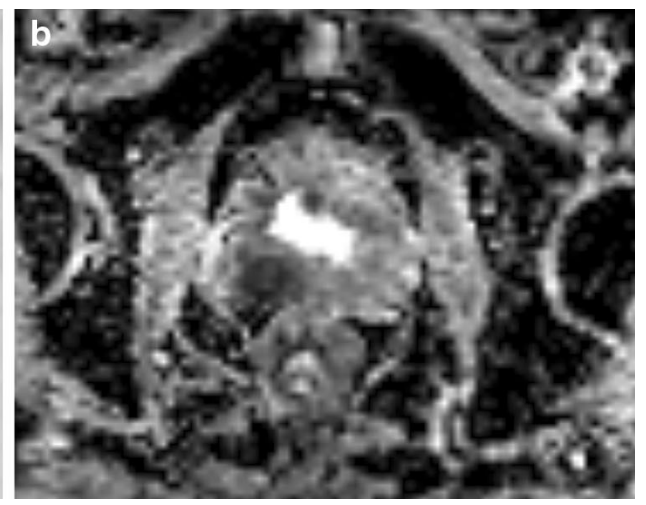

inverted. Transurethral resection of the prostate changes are present. b Quantitative ADC measurements can also obtained; right mid-gland PCA shows dark signal on ADC image

Similar to Doppler US, the MRI correlate for CEUS is DCE-MRI. While Doppler US primarily assesses macrovessels, CEUS is advantageous because the small size of the microbubbles allows evaluation of the 
microvasculature [9]. CEUS images are dynamically acquired allowing measurement and quantification of tissue perfusion as a time-intensity curve analogous to the kinetic curve of DCE-MRI [44]. However unlike gadolinium contrast, CEUS microbubbles remain entirely within the intravascular space, potentially providing additional complementary information to DCE-MRI $[9,44]$.

\section{Future}

Use of B-mode and Doppler US is well established in prostate imaging, but the additional modalities of USE and CEUS show promise in improving PCA detection and diagnosis. Discrepancies in prior study results may be due to heterogeneity of study design and population selection, and large prospective multicenter studies are warranted for validation. 3D methods of USE and CEUS are becoming available and would facilitate accuracy of fusion across modalities, notably with mpMRI. Currently prostate USE and MRI/TRUS fusion are not available on the same transducer; development of this may improve accuracy by allowing real-time USE visualization of prostate lesions identified on mpMRI during biopsy. Finally, use of artificial neural network analysis on prostate images has shown benefit in improving PCA detection using B-mode images [48], and the same principles may extend to the other US modalities.

\section{Conclusion}

Imaging plays a critical role in the fast changing landscape of PCA detection and diagnosis. Here we summarize the use of US in prostate imaging along with their MRI correlates and shared physiologic bases. The complementary advantages and rapid development of US technologies will undoubtedly lead to increased use of US in conjunction with MRI in the future of PCA management.

\section{Compliance with Ethical Guidelines}

Conflict of Interest Joy Liau, Daniel Goldberg, and Hina Arif-Tiwari each declare no potential conflicts of interest.

Human and Animal Rights This article does not contain any studies with human or animal subjects performed by any of the authors.

Open Access This article is distributed under the terms of the Creative Commons Attribution 4.0 International License (http:// creativecommons.org/licenses/by/4.0/), which permits unrestricted use, distribution, and reproduction in any medium, provided you give appropriate credit to the original author(s) and the source, provide a link to the Creative Commons license, and indicate if changes were made.

\section{References}

Recently published papers of particular interest have been highlighted as:

- Of importance

•- Of major importance

1. Siegel RL, Miller KD, Jemal A. Cancer statistics, 2018. CA Cancer J Clin. 2018;68(1):7-30.

2. - Tyloch JF, Wieczorek AP. The standards of an ultrasound examination of the prostate gland. Part 2. J Ultrason. 2017;17(68):43-58. This paper reviews the technique and clinical applications of ultrasound to prostate disease detection and management.

3. Borza T, Konijeti R, Kibel AS. Early detection, PSA screening, and management of overdiagnosis. Hematol Oncol Clin North Am. 2013;27(6):1091-110.

4. •- Barr RG, Cosgrove D, Brock M, Cantisani V, Correas JM, Postema AW, et al. WFUMB guidelines and recommendations on the clinical use of ultrasound elastography: part 5. prostate. Ultrasound Med Biol. 2017;43(1):27-48. This paper provides a comprehensive review of and consensus guidelines for prostate ultrasound elastography.

5. Li Q, Xiang F, Lin X, Grajo JR, Yang L, Xu Y, et al. The role of imaging in prostate cancer care pathway: novel approaches to urologic management challenges along 10 imaging touch points. Urology. 2018;119:23-31.

6. Boesen L, Norgaard N, Logager V, Balslev I, Thomsen HS. Where do transrectal ultrasound- and magnetic resonance imaging-guided biopsies miss significant prostate cancer? Urology. 2017;110:154-60.

7. Stark JR, Perner S, Stampfer MJ, Sinnott JA, Finn S, Eisenstein AS, et al. Gleason score and lethal prostate cancer: does $3+4=4+3$ ? J Clin Oncol. 2009;27(21):3459-64.

8. Schoots IG, Roobol MJ, Nieboer D, Bangma CH, Steyerberg EW, Hunink MG. Magnetic resonance imaging-targeted biopsy may enhance the diagnostic accuracy of significant prostate cancer detection compared to standard transrectal ultrasound-guided biopsy: a systematic review and meta-analysis. Eur Urol. 2015;68(3):438-50.

9. Sarkar S, Das S. A review of imaging methods for prostate cancer detection. Biomed Eng Comput Biol. 2016;7(Suppl 1):1-15.

10. - Gold SA, Hale GR, Bloom JB, Smith CP, Rayn KN, Valera V, et al. Follow-up of negative MRI-targeted prostate biopsies: when are we missing cancer? World J Urol. 2018. This paper discusses the important problem of false negative biopsies of prostate lesions identified on multiparametric MRI and biopsied by MRI and transrectal ultrasound fusion.

11. Rouviere O, Melodelima C, Hoang Dinh A, Bratan F, Pagnoux G, Sanzalone T, et al. Stiffness of benign and malignant prostate tissue measured by shear-wave elastography: a preliminary study. Eur Radiol. 2017;27(5):1858-66.

12. Ozgur A, Onol FF, Turkeri LN. Important preoperative prognostic factors for extracapsular extension, seminal vesicle invasion and lymph node involvement in cases with radical retropubic prostatectomy. Int Urol Nephrol. 2004;36(3):369-73.

13. Fregene TA, Khanuja PS, Noto AC, Gehani SK, Van Egmont EM, Luz DA, et al. Tumor-associated angiogenesis in prostate cancer. Anticancer Res. 1993;13(6b):2377-81.

14. Halpern EJ, Frauscher F, Strup SE, Nazarian LN, O'Kane P, Gomella LG. Prostate: high-frequency Doppler US imaging for cancer detection. Radiology. 2002;225(1):71-7. 
15. Okihara K, Kojima M, Nakanouchi T, Okada K, Miki T. Transrectal power Doppler imaging in the detection of prostate cancer. BJU Int. 2000;85(9):1053-7.

16. Sauvain JL, Sauvain E, Rohmer P, Louis D, Nader N, Papavero $\mathrm{R}$, et al. Value of transrectal power Doppler sonography in the detection of low-risk prostate cancers. Diagn Interv Imaging. 2013;94(1):60-7.

17. Harvey $\mathrm{H}$, deSouza NM. The role of imaging in the diagnosis of primary prostate cancer. J Clin Urol. 2016;9(2 Suppl):11-7.

18. Sigrist RMS, Liau J, Kaffas AE, Chammas MC, Willmann JK. Ultrasound elastography: review of techniques and clinical applications. Theranostics. 2017;7(5):1303-29.

19. Tuxhorn JA, Ayala GE, Smith MJ, Smith VC, Dang TD, Rowley DR. Reactive stroma in human prostate cancer: induction of myofibroblast phenotype and extracellular matrix remodeling. Clin Cancer Res. 2002;8(9):2912-23.

20. Tsutsumi M, Miyagawa T, Matsumura T, Endo T, Kandori S, Shimokama T, et al. Real-time balloon inflation elastography for prostate cancer detection and initial evaluation of clinicopathologic analysis. Am J Roentgenol. 2010;194(6):W471-6.

21. Pallwein L, Mitterberger M, Struve P, Pinggera G, Horninger W, Bartsch G, et al. Real-time elastography for detecting prostate cancer: preliminary experience. BJU Int. 2007;100(1):42-6.

22. Brock M, von Bodman C, Sommerer F, Loppenberg B, Klein T, Deix $\mathrm{T}$, et al. Comparison of real-time elastography with greyscale ultrasonography for detection of organ-confined prostate cancer and extra capsular extension: a prospective analysis using whole mount sections after radical prostatectomy. BJU Int. 2011;108(8 Pt 2):E217-22.

23. Kamoi K, Okihara K, Ochiai A, Ukimura O, Mizutani Y, Kawauchi A, et al. The utility of transrectal real-time elastography in the diagnosis of prostate cancer. Ultrasound Med Biol. 2008;34(7):1025-32.

24. Xu G, Feng L, Yao M, Wu J, Guo L, Yao X, et al. A new 5-grading score in the diagnosis of prostate cancer with real-time elastography. Int J Clin Exp Pathol. 2014;7(7):4128-35.

25. Ma Q, Yang DR, Xue BX, Wang C, Chen HB, Dong Y, et al. Transrectal real-time tissue elastography targeted biopsy coupled with peak strain index improves the detection of clinically important prostate cancer. Oncol Lett. 2017;14(1):210-6.

26. Ferrari FS, Scorzelli A, Megliola A, Drudi FM, Trovarelli S, Ponchietti R. Real-time elastography in the diagnosis of prostate tumor. J Ultrasound. 2009;12(1):22-31.

27. Brock M, von Bodman C, Palisaar RJ, Loppenberg B, Sommerer $\mathrm{F}$, Deix $\mathrm{T}$, et al. The impact of real-time elastography guiding a systematic prostate biopsy to improve cancer detection rate: a prospective study of 353 patients. J Urol. 2012;187(6):2039-43.

28. Pallwein L, Mitterberger M, Struve P, Horninger W, Aigner F, Bartsch $\mathrm{G}$, et al. Comparison of sonoelastography guided biopsy with systematic biopsy: impact on prostate cancer detection. Eur Radiol. 2007;17(9):2278-85.

29. Wang R, Chen JJ, Hu B. Transrectal real-time elastographyguided transperineal prostate biopsy as an improved tool for prostate cancer diagnosis. Int J Clin Exp Med. 2015;8(4):6522-9.

30. Salomon G, Drews N, Autier P, Beckmann A, Heinzer H, Hansen $\mathrm{J}$, et al. Incremental detection rate of prostate cancer by real-time elastography targeted biopsies in combination with a conventional 10-core biopsy in 1024 consecutive patients. BJU Int. 2014;113(4):548-53.

31. Schiffmann J, Grindei M, Tian Z, Yassin DJ, Steinwender T, Leyh-Bannurah SR, et al. Limitations of elastography based prostate biopsy. J Urol. 2016;195(6):1731-6.

32. Sumura M, Shigeno K, Hyuga T, Yoneda T, Shiina H, Igawa M. Initial evaluation of prostate cancer with real-time elastography based on step-section pathologic analysis after radical prostatectomy: a preliminary study. Int J Urol. 2007;14(9):811-6.
33. Pelzer AE, Heinzelbecker J, Weiss C, Fruhbauer D, Weidner AM, Kirchner M, et al. Real-time sonoelastography compared to magnetic resonance imaging using four different modalities at 3.0 $\mathrm{T}$ in the detection of prostate cancer: strength and weaknesses. Eur J Radiol. 2013;82(5):814-21.

34. Brock M, Loppenberg B, Roghmann F, Pelzer A, Dickmann M, Becker $\mathrm{W}$, et al. Impact of real-time elastography on magnetic resonance imaging/ultrasound fusion guided biopsy in patients with prior negative prostate biopsies. J Urol. 2015;193(4):1191-7.

35. Mousavi SR, Rivaz H, Czarnota GJ, Samani A, Sadeghi-Naini A. Ultrasound elastography of the prostate using an unconstrained modulus reconstruction technique: a pilot clinical study. Transl Oncol. 2017;10(5):744-51.

36. Junker D, Schafer G, Aigner F, Schullian P, Pallwein-Prettner L, Bektic J, et al. Potentials and limitations of real-time elastography for prostate cancer detection: a whole-mount step section analysis. Sci World J. 2012;2012:193213.

37. Aigner F, Pallwein L, Junker D, Schafer G, Mikuz G, Pedross F, et al. Value of real-time elastography targeted biopsy for prostate cancer detection in men with prostate specific antigen $1.25 \mathrm{ng} / \mathrm{ml}$ or greater and $4.00 \mathrm{ng} / \mathrm{ml}$ or less. J Urol. 2010;184(3):913-7.

38. Woo S, Kim SY, Cho JY, Kim SH. Shear wave elastography for detection of prostate cancer: a preliminary study. Korean J Radiol. 2014;15(3):346-55.

39. Correas JM, Tissier AM, Khairoune A, Vassiliu V, Mejean A, Helenon O, et al. Prostate cancer: diagnostic performance of realtime shear-wave elastography. Radiology. 2015;275(1):280-9.

40. Boehm K, Salomon G, Beyer B, Schiffmann J, Simonis K, Graefen M, et al. Shear wave elastography for localization of prostate cancer lesions and assessment of elasticity thresholds: implications for targeted biopsies and active surveillance protocols. J Urol. 2015;193(3):794-800.

41. Wei C, Li C, Szewczyk-Bieda M, Upreti D, Lang S, Huang Z, et al. Performance characteristics of transrectal shear wave elastography imaging in the evaluation of clinically localized prostate cancer: a prospective study. J Urol. 2018.

42. Porsch M, Gorner C, Wendler JJ, Liehr UB, Lux A, Siedentopf S, et al. Inability of shear-wave elastography to distinguish malignant from benign prostate tissue-a comparison of biopsy, wholemount sectioning and shear-wave elastography. J Ultrason. 2016;16(67):348-58.

43. Boehm K, Budaus L, Tennstedt P, Beyer B, Schiffmann J, Larcher A, et al. Prediction of significant prostate cancer at prostate biopsy and per core detection rate of targeted and systematic biopsies using real-time shear wave elastography. Urol Int. 2015;95(2):189-96.

44. Tang MX, Mulvana H, Gauthier T, Lim AK, Cosgrove DO, Eckersley RJ, et al. Quantitative contrast-enhanced ultrasound imaging: a review of sources of variability. Interface Focus. 2011;1(4):520-39.

45. Yunkai Z, Yaqing C, Jun J, Tingyue Q, Weiyong L, Yuehong Q, et al. Comparison of contrast-enhanced ultrasound targeted biopsy versus standard systematic biopsy for clinically significant prostate cancer detection: results of a prospective cohort study with 1024 patients. World J Urol. 2018.

46. Koh J, Jung DC, Oh YT, Yoo MG, Noh S, Han KH, et al. Additional targeted biopsy in clinically suspected prostate cancer: prospective randomized comparison between contrast-enhanced ultrasound and sonoelastography guidance. Ultrasound Med Biol. 2015;41(11):2836-41.

47. Li Y, Tang J, Fei X, Gao Y. Diagnostic performance of contrast enhanced ultrasound in patients with prostate cancer: a metaanalysis. Acad Radiol. 2013;20(2):156-64. 
48. Loch T. Computerized transrectal ultrasound (C-TRUS) of the prostate: detection of cancer in patients with multiple negative systematic random biopsies. World J Urol. 2007;25(4):375-80.
Publisher's Note Springer Nature remains neutral with regard to jurisdictional claims in published maps and institutional affiliations. 